\title{
NONLINEAR DIFFUSIVE PHENOMENA OF ENTROPY WEAK SOLUTIONS FOR A SYSTEM OF QUASILINEAR HYPERBOLIC CONSERVATION LAWS WITH DAMPING
}

\author{
BY \\ L. HSIAO AND T. LUO \\ Institute of Mathematics, Academia Sinica, Beijing
}

\begin{abstract}
We consider a system of isentropic flow through porous media and show that nonlinear diffusive phenomena occur even if there are shocks in the flow.
\end{abstract}

1. Introduction. We study the following hyperbolic conservation laws with damping:

$$
\left\{\begin{array}{l}
v_{t}-u_{x}=0, \\
u_{t}+p(v)_{x}=-\alpha u, \quad \alpha>0, \quad p^{\prime}(v)<0 \text { for } v>0,
\end{array}\right.
$$

which can be viewed as isentropic Euler equations in Lagrangian coordinates with friction term, $-\alpha u$, for the momentum equation and can be used to model the compressible flow through porous media where $u$ is the velocity, $v>0$ is the specific volume, and $p(v)$ is the pressure.

The well-known porous media equation can be obtained by approximating the second equation with Darcy's law:

$$
\left\{\begin{array}{l}
v_{t}=-\frac{1}{\alpha} p(v)_{x x} \\
p(v)_{x}=-\alpha u
\end{array}\right.
$$

The diffusion effect created by the damping is studied for solutions of (1.1) without shock waves in [HLI1, HLI2], which shows that the solutions of (1.1) tend to those of (1.2) as the time $t$ tends to infinity. Namely, nonlinear diffusive phenomena in the solutions of (1.1) are discovered for solutions of (1.1) without shock waves. This result has been established even for adiabatic flow (see [HLU]). In the case when shock waves may develop in the solution of (1.1) with rough data, it is expected in [HLI1] that (1.2) would still model the time-asymptotic behavior of (1.1).

Received April 17, 1995.

1991 Mathematics Subject Classification. Primary 35, 76.

Project supported by the National Natural Science Foundation of China.

(C)1998 Brown University 
The purpose of this paper is to prove this expectation, which means that Darcy's law may be obtained, in fact, from the more complete equations (1.1) time-asymptotically even in the presence of shocks.

For simplicity, we consider (1.1) with Riemann data, namely

$$
(u(x, 0), v(x, 0))= \begin{cases}\left(u_{-}, v_{-}\right) & \text {for } x<0, \\ \left(u_{+}, v_{+}\right) & \text {for } x>0,\end{cases}
$$

where $v_{\mp}>0$, and we take a typical form of the state function $p(v)$ to discuss, instead of a general one, namely $p(v)=a v^{-\gamma}$ with $a>0$ and $1<\gamma<3$, which is the state function for polytropic gas. Without loss of generality, this paper only deals with the case in which the two states $\left(u_{-}, v_{-}\right)$and $\left(u_{+}, v_{+}\right)$are connected by a forward shock curve in the phase plane and the entropy condition is satisfied as well, namely

$$
\left\{\begin{array}{l}
u_{+}-u_{-}=-\left(v_{+}-v_{-}\right) \sqrt{\frac{p\left(v_{+}\right)-p\left(v_{-}\right)}{v_{-}-v_{+}}}, \\
v_{+}>v_{-} .
\end{array}\right.
$$

For the system (1.1) without the damping term, i.e., $\alpha=0$, a Riemann problem has been studied very well (see $[\mathrm{CH}],[\mathrm{DD}],[\mathrm{LI}],[\mathrm{SM}]$, etc.). However, for the system (1.1) with the damping term, the Riemann problem is much more complicated since there is no self-similar solution anymore and has not been studied so well yet in the literature although the problem is significant in the qualitative theory of inhomogeneous hyperbolic systems and in the applications as well.

It is known from [HT1, HT2] that if the initial shock is suitably weak, then the problem of (1.1), (1.3) with (1.4) has a unique entropy weak solution globally in time that is piecewise smooth and contains a forward shock $x=x_{2}(t)(0 \leq t<+\infty)$, satisfying the entropy condition, namely,

$$
\left\{\begin{array}{l}
\frac{d x_{2}(t)}{d t}=\sqrt{\frac{p\left(v_{R}\right)-p\left(v_{L}\right)}{v_{L}-v_{R}}}, \quad x_{2}(0)=0, \\
u_{R}-u_{L}=-\left(v_{R}-v_{L}\right) \cdot \sqrt{\frac{p\left(v_{R}\right)-p\left(v_{L}\right)}{v_{L}-v_{R}}} \\
v_{R}>v_{L},
\end{array}\right.
$$

where $\left(u_{R}, v_{R}\right)=(u, v)\left(x_{2}(t)+0, t\right),\left(u_{L}, v_{L}\right)=(u, v)\left(x_{2}(t)-0, t\right)$. Moreover, it is proved in $[\mathrm{HT} 1, \mathrm{HT} 2]$ that

(i) the solution $(u, v)$ is smooth in the domains $\Omega_{1}$ and $\Omega_{2}$ up to the boundary respectively, where

$$
\begin{aligned}
& \Omega_{1}=:\left\{(x, t): x<x_{2}(t), t \geq 0\right\}, \\
& \Omega_{2}=:\left\{(x, t): x>x_{2}(t), t \geq 0\right\} ;
\end{aligned}
$$

(ii) in the domain $\Omega_{2},(u, v)$ is defined as

$$
(u, v)=\left\{u^{+}(x, t), v^{+}(x, t)\right\}=\left\{u_{+} e^{-t}, v_{+}\right\},
$$

and in the domain $I_{1},(u, v)$ is defined as

$$
(u, v)=\left\{u^{-}(x, t), v^{-}(x, t)\right\}=\left\{u_{-} e^{-t}, v_{-}\right\}
$$


where $I_{1}=:\left\{(x, t): x \leq x_{1}(t), t \geq 0\right\}$ and $x_{1}(t)$ is the backward characteristic satisfying

$$
\frac{d x_{1}(t)}{d t}=\lambda\left(v_{-}\right)=-\sqrt{\gamma}\left(v_{-}\right)^{-\frac{\gamma+1}{2}}, \quad x_{1}(0)=0
$$

(iii) along the shock curve $x=x_{2}(t)$,

$$
\left(v_{+}-v_{-}\right) e^{-A_{1} t} \leq v_{+}-v \leq\left(v_{+}-v_{-}\right) e^{-A_{2} t}, \quad t \geq 0,
$$

where $v=v\left(x_{2}(t)-0, t\right), A_{i}$ is a positive constant $(i=1,2)$, and

(iv) $v_{-} \leq v(x, t) \leq v_{+}, \quad$ for $x \in R, t \geq 0$.

On the other hand, it is well known that (1.2) has a unique similarity solution $v^{*}(\eta)$, $\eta=\frac{x}{\sqrt{t+1}}$, satisfying $v^{*}( \pm \infty)=v_{ \pm}$and $v^{*}(\eta)$ is strictly monotone for $\eta \in R$ (see [DP]).

The main result obtained in the present paper says that there exists an $\bar{x}_{0} \in R$ such that if $\delta=\left|u_{+}-u_{-}\right|+\left|v_{+}-v_{-}\right|$is suitably small, then

$$
\begin{aligned}
\sup _{x \in R}\left|u(x, t)-u^{*}\left(x+\bar{x}_{0}, t\right)\right|+\sup _{x \in R} \mid v(x, t)-v^{*} & \left(x+\bar{x}_{0}, t\right) \mid \\
& =O(1)(t+1)^{-1 / 2}, \quad \text { as } t \rightarrow+\infty
\end{aligned}
$$

where

$$
v^{*}\left(x+\bar{x}_{0}, t\right)=v^{*}\left(\frac{x+\bar{x}_{0}}{\sqrt{t+1}}\right), \quad u^{*}\left(x+\bar{x}_{0}, t\right)=\frac{-1}{\alpha} p\left(v^{*}\left(x+\bar{x}_{0}, t\right)\right)_{x} .
$$

This shows that nonlinear diffusive phenomena also occur for entropy weak solutions of (1.1).

2. Estimates along the shock curve $x=x_{2}(t)$. We deduce certain decay estimates along the shock curve $x=x_{2}(t)$ in this section, which plays a key role for establishing the main result in the next section.

Consider the system (1.1) with $a=1$ and $\alpha=1$, for convenience, namely

$$
\left\{\begin{array}{l}
v_{t}-u_{x}=0 \\
u_{t}+p(v)_{x}=-u, \quad p(v)=v^{-\gamma} \quad(1<\gamma<3) .
\end{array}\right.
$$

It is easy to observe that the characteristic speed of (2.1) takes the form

$$
\lambda=-\sqrt{\gamma} v^{-\frac{\gamma+1}{2}} \text { and } \mu=\sqrt{\gamma} v^{-\frac{\gamma+1}{2}}
$$

respectively, corresponding to the backward and forward family.

Introduce Riemann invariants

$$
\begin{aligned}
& r=u-\frac{2 \sqrt{\gamma}}{\gamma-1} v^{\frac{1-\gamma}{2}} \\
& s=u+\frac{2 \sqrt{\gamma}}{\gamma-1} v^{\frac{1-\gamma}{2}}
\end{aligned}
$$

Thus

$$
\begin{aligned}
& u=\frac{s+r}{2} \\
& v=\left[\frac{\gamma-1}{4 \sqrt{\gamma}}(s-r)\right]^{\frac{2}{1-\gamma}},
\end{aligned}
$$


and the system (2.1) can be written as

$$
\begin{aligned}
& r_{t}+\lambda r_{x}=-\frac{1}{2}(r+s), \\
& s_{t}+\mu s_{x}=-\frac{1}{2}(r+s),
\end{aligned}
$$

which is equivalent to (2.1) wherever the solution is smooth.

By the notation

$$
\begin{aligned}
& { }^{\prime}=\frac{\partial}{\partial t}+\lambda \frac{\partial}{\partial x} \\
& { }^{\prime}=\frac{\partial}{\partial t}+\mu \frac{\partial}{\partial x}
\end{aligned}
$$

Eqs. (2.4) become

$$
\begin{aligned}
& r^{\prime}=-\frac{1}{2}(r+s), \\
& s^{\prime}=-\frac{1}{2}(r+s) .
\end{aligned}
$$

It is known from [HT1, HT2] that, along the shock curve $x=x_{2}(t)(t \geq 0)$,

$$
\begin{gathered}
{\left[\sqrt{\gamma} v^{-\frac{1+\gamma}{2}}+\sqrt{\frac{v^{-\gamma}-v_{+}^{-\gamma}}{v_{+}-v}}\right]\left\{1+\frac{\left[\gamma v^{-(1+\gamma)} \cdot\left(v_{+}-v\right)+\left(v^{-\gamma}-v_{+}^{-\gamma}\right)\right]}{2 \sqrt{\gamma\left(v_{+}-v\right)\left(v^{-\gamma}-v_{+}^{-\gamma}\right)}} v^{\frac{1+\gamma}{2}}\right\} r_{x}} \\
=2 \sqrt{\left(v_{+}-v\right)\left(v^{-\gamma}-v_{+}^{-\gamma}\right)}-\left[\sqrt{\gamma} v^{-\frac{1+\gamma}{2}}-\sqrt{\frac{v^{-\gamma}-v_{+}^{-\gamma}}{v_{+}-v}}\right] \\
\cdot\left\{-1+\frac{\left[\gamma v^{-(1+\gamma)} \cdot\left(v_{+}-v\right)+\left(v^{-\gamma}-v_{+}^{-\gamma}\right)\right]}{2 \sqrt{\gamma\left(v_{+}-v\right)\left(v^{-\gamma}-v_{+}^{-\gamma}\right)}} \cdot v^{\frac{1+\gamma}{2}}\right\} \cdot s_{x},
\end{gathered}
$$

where $r_{x}, s_{x}$, and $v$ take values at $\left(x_{2}(t)-0, t\right)$, and it is shown in [HT1, HT2] that

$$
\left|s_{x}\left(x_{2}(t)-0, t\right)\right| \leq C ;
$$

hereafter $C$ denotes a generic constant, independent of $t$.

Due to (1.8), it follows that, along $x=x_{2}(t)$,

$$
v_{+}-\delta e^{-A_{2} t} \leq v\left(x_{2}(t)-0, t\right) \leq v_{+}+\delta e^{-A_{1} t}
$$

Conditions (2.7)-(2.9), with the help of the mean value theorem used on $\left(v^{-\gamma}-v_{+}^{-\gamma}\right)$, imply that

$$
\left|r_{x}\left(x_{2}(t)-0, t\right)\right| \leq C e^{-\beta_{1} t}, \quad t \geq 0 \text { for some } \beta_{1}>0 .
$$

To get the decay estimates of $s_{x}$ along $x=x_{2}(t)$, we need the following lemma.

LEMMA 2.1. If $\delta=\left|u_{+}-u_{-}\right|+\left|v_{+}-v_{-}\right|$is suitably small, then

$$
\left|s_{x x}(x, t)\right| \leq C \quad \text { for } x<x_{2}(t), t \geq 0 \text {. }
$$

Particularly,

$$
\left|s_{x x}\left(x_{2}(t)-0, t\right)\right| \leq C, \quad t \geq 0 .
$$


Proof. The existence of all of the derivatives $r_{x x}, s_{x x}, r_{x x x}, r_{x x t}, s_{x x x}, s_{x x t}$, etc., in the domain $\Omega_{1}=\left\{(x, t): x \leq x_{2}(t), t \geq 0\right\}$ can be obtained, based on (2.4), by using difference quotients and then taking limits.

Let

$$
y=\mu^{1 / 2} s_{x}+\frac{g}{2} \quad \text { for }(x, t) \in \Omega_{1},
$$

where

$$
g=\frac{4 \gamma^{\frac{1}{4}}}{3-\gamma} v^{\frac{3-\gamma}{4}} .
$$

With the help of (2.4), it is easy to find that

$$
\begin{aligned}
y_{t}+ & \sqrt{\gamma} \cdot v^{-\frac{\gamma+1}{2}} y_{x} \\
& =-f\left(y-\frac{1}{2} g\right)\left(y-\frac{\gamma-1}{\gamma+1} g\right) \quad \text { for }(x, t) \in \Omega_{1}
\end{aligned}
$$

where $f=\frac{\gamma+1}{3-\gamma} g^{-1}$.

Differentiating (2.14) with respect to $x$, we arrive, by virtue of $(2.2),(2.3),(2.4)$, and (2.6), at

$$
\begin{aligned}
\left(y_{x}\right)^{\prime} & -\frac{(\gamma+1)}{4} v^{-1} \cdot v^{\prime} \cdot y_{x}+\frac{(\gamma+1)}{4} v^{-1} s_{x} y_{x} \\
& =f_{1}(x, t)-f\left[2 y-\frac{(3 \gamma-1)}{2(\gamma+1)} g\right] y_{x}+f_{2}(x, t) \quad \text { for }(x, t) \in \Omega_{1},
\end{aligned}
$$

where

$$
\begin{aligned}
& f_{1}(x, t)=-f_{x}\left[y^{2}-\frac{(2 \gamma-1)}{2(\gamma+1)} g y+\frac{\gamma-1}{2(\gamma+1)} g^{2}\right](x, t), \\
& f_{2}(x, t)=-f\left[-\frac{(3 \gamma-1)}{2(\gamma+1)} g_{x} \cdot y+\frac{(\gamma-1)}{(\gamma+1)} g \cdot g_{x}\right](x, t) .
\end{aligned}
$$

Then, multiplying $(2.15)$ by $v^{-\frac{\gamma+1}{4}}$, we get

$$
\begin{aligned}
& \left(v^{-\frac{\gamma+1}{4}} y_{x}\right)^{\prime} \\
& \quad=-\left\{f\left[2 y-\frac{(3 \gamma-1)}{2(\gamma+1)} g\right]+\frac{(\gamma+1)}{4} v^{-1} s_{x}\right\} \cdot\left(v^{-\frac{\gamma+1}{4}} \cdot y_{x}\right)+f_{3}(x, t), \quad(x, t) \in \Omega_{1},
\end{aligned}
$$

where

$$
f_{3}(x, t)=v^{-\frac{\gamma+1}{4}}\left[f_{1}(x, t)+f_{2}(x, t)\right] .
$$

Let

$$
b=f\left[2 y-\frac{(3 \gamma-1)}{2(\gamma+1)} g\right]+\frac{(\gamma+1)}{4} \cdot v^{-1} s_{x}, \quad(x, t) \in \Omega_{1} .
$$

By the definition of $y, f$, and $g$, it is easy to see that

$$
b(x, t)=\frac{1}{2}+\frac{3(\gamma+1)}{4}\left(v^{-1} s_{x}\right)(x, t), \quad(x, t) \in \Omega_{1} .
$$


It is known from [HT1, HT2] that $\left|s_{x}\right|$ is small if $\delta$ is small. This implies that

$$
b(x, t) \geq \frac{1}{4} \quad \text { for }(x, t) \in \Omega_{1}
$$

provided $\delta$ is suitably small.

We also know from [HT1, HT2] that

$$
\left|f_{3}(x, t)\right| \leq C \quad \text { for }(x, t) \in \Omega_{1} .
$$

For any fixed $(x, t) \in \Omega_{1}$, let $x_{2}(\tau ; x, t)(0 \leq \tau \leq t)$ denote the forward characteristic passing through $(x, t)$ such that $x_{2}(t ; t, x)=x$ and $x_{2}(0 ; x, t)=\beta$. Let

$$
k(\tau)=v^{-\frac{\gamma+1}{4}} y_{x}\left(x_{2}(\tau ; x, t), \tau\right)
$$

where $(x, t) \in \Omega_{1}$ is fixed. We still use the same notations $b$ and $f_{3}$ to denote the corresponding values taken along $x_{2}(\tau ; x, t)$ respectively, namely,

$$
\begin{aligned}
b(\tau) & =b\left(x_{2}(\tau ; x, t), \tau\right), \\
f_{3}(\tau) & =f_{3}\left(x_{2}(\tau ; x, t), \tau\right) .
\end{aligned}
$$

Then, (2.16) implies that

$$
\frac{d k(\tau)}{d \tau}=-b(\tau) k(\tau)+f_{3}(\tau), \quad 0 \leq \tau \leq t
$$

where it is known from (2.18) that

$$
\left|f_{3}(\tau)\right| \leq C, \quad 0 \leq \tau \leq t .
$$

Multiply $(2.19)$ by $\exp \left[\int_{0}^{\tau} b(\xi) d \xi\right]$ and integrate the resulting equation over $[0, t]$. It turns out from (2.17)-(2.19) that

$$
|k(\tau)| \leq C, \quad 0 \leq \tau \leq t
$$

Particularly,

$$
|k(t)| \leq C
$$

Due to the arbitrariness of $(x, t) \in \Omega_{1}$, we obtain

$$
\left|v^{-\frac{\gamma+1}{4}} y_{x}(x, t)\right| \leq C \quad \text { for }(x, t) \in \Omega_{1} \text {. }
$$

Lemma 2.1 follows then by virtue of the facts: $v_{-} \leq v(x, t) \leq v_{+}$, the uniform boundedness of $u_{x}$ and $v_{x}$ in $\Omega_{1}$ (see [HT1, HT2]), and the definition of $y$.

The decay estimate of $s_{x}$ along the shock curve $x=x_{2}(t)$ is given in the next lemma.

LEMMA 2.2. If $\delta$ is suitably small, then it follows that

$$
\left|s_{x}\left(x_{2}(t)-0, t\right)\right| \leq C e^{-\beta_{2} t}, \quad t \geq 0, \quad \text { for some } \beta_{2}>0 .
$$

Proof. Let

$$
y_{1}\left(x_{2}(t), t\right)=:\left[\mu\left(v_{+}\right)\right]^{1 / 2} \cdot \frac{2 v_{+}}{3-\gamma}-\left(\mu^{1 / 2} s_{x}+\frac{g}{2}\right)\left(x_{2}(t)-0, t\right), \quad t \geq 0
$$

and

$$
Y(t)=: y_{1}\left(x_{2}(t), t\right)
$$

where $\mu\left(v_{+}\right)$is the value of $\mu(v)$ at $v=v_{+}$. 
It is easy to verify that

$$
\begin{aligned}
\frac{d Y(t)}{d t}= & f\left[\mu\left(v_{+}\right)^{1 / 2} \frac{2 v_{+}}{3-\gamma}-\frac{1}{2} g-Y(t)\right]\left[\mu\left(v_{+}\right)^{1 / 2} \frac{2 v_{+}}{3-\gamma}-\frac{\gamma-1}{\gamma+1} g-Y(t)\right] \\
& +y_{1 x}\left(x_{2}(t)-0, t\right)\left[\frac{d x_{2}(t)}{d t}-\mu\left(v\left(x_{2}(t)-0, t\right)\right)\right]
\end{aligned}
$$

where $g$ and $f$ take values at $v=v\left(x_{2}(t)-0, t\right)$, defined by (2.13) and (2.14) respectively.

We estimate next each term on the right-hand side of $(2.21)$.

Define

$$
k_{1}(t)=: \mu\left(v_{+}\right)^{1 / 2} \frac{2 v_{+}}{3-\gamma}-\frac{1}{2} g\left(x_{2}(t)-0, t\right)
$$

It follows from (1.8) and (2.13) that

$$
\left|k_{1}(t)\right| \leq C e^{-\beta_{3} t}, t \geq 0, \quad \text { for some } \beta_{3}>0 .
$$

Let

$$
\begin{aligned}
k_{2}(t) & =: \mu\left(v_{+}\right)^{1 / 2} \frac{2 v_{+}}{3-\gamma}-\frac{\gamma-1}{\gamma+1} g-Y(t) \\
& =\left(\mu^{1 / 2} s_{x}+\frac{3-\gamma}{2(\gamma+1)} g\right)\left(x_{2}(t)-0, t\right) .
\end{aligned}
$$

Since $\left|s_{x}\right|$ is small when $\delta$ is small (see [HT1, HT2]), we are able to choose a positive constant $a_{1}>0$ such that

$$
k_{2}(t) \geq a_{1}, \quad t \geq 0
$$

Define

$$
k_{3}(t)=: y_{1 x}\left(x_{2}(t)-0, t\right)\left[\frac{d x_{2}(t)}{d t}-\mu\left(v\left(x_{2}(t)-0, t\right)\right)\right] .
$$

Due to (1.5), (1.8), Lemma 2.1, and the uniform boundedness of $\left(u_{x}, v_{x}\right)\left(x_{2}(t)-0, t\right)$ for $t \geq 0$ (see [HT1, HT2]), it can be shown that

$$
\left|k_{3}(t)\right| \leq C e^{-\beta_{4} t}, t \geq 0, \quad \text { for some } \beta_{4}>0 .
$$

In terms of $k_{1}(t), k_{2}(t)$, and $k_{3}(t),(2.21)$ can be written as

$$
\frac{d Y(t)}{d t}=-f k_{2}(t) Y(t)+f k_{1}(t) k_{2}(t)+k_{3}(t), \quad t \geq 0
$$

Conditions (2.22)-(2.24), with the definition of $f$ together, yield

$$
f k_{2}(t) \geq d_{0}>0 \quad(t \geq 0), \quad \text { for some positive constant } d_{0}>0
$$

and

$$
\left|f k_{1}(t) k_{2}(t)+k_{3}(t)\right| \leq C e^{-\beta_{5} t}(t \geq 0) \text { for some positive constant } \beta_{5}>0 .
$$

Multiplying (2.25) by $\exp \left[\int_{0}^{t}\left(f k_{2}\right)(\xi) d \xi\right]$ and integrating it with respect to $t$, we arrive, with the help of (2.25)-(2.27), at

$$
|Y(t)| \leq C e^{-\beta_{6} t}(t \geq 0) \text { for some positive constant } \beta_{6}>0 \text {. }
$$


It is clear from (1.8) that

$$
\left|\mu\left(v_{+}\right)^{1 / 2} \frac{2 v_{+}}{3-\gamma}-\frac{g}{2}\left(x_{2}(t)-0, t\right)\right| \leq C e^{-\beta_{7} t} \quad(t \geq 0)
$$

for some positive constant $\beta_{7}>0$.

Lemma 2.2 follows then from (2.28), (2.29), and the definition of $Y$.

Differentiating (2.7) with respect to $t$ along $x=x_{2}(t)$, and using (2.4), we can find a relation between $r_{x x}$ and $s_{x x}$. This, combined with Lemma 2.1 and the uniform boundedness of $r_{x}, s_{x}, r_{t}$, and $s_{t}$, yields that

$$
\left|r_{x x}\left(x_{2}(t)-0, t\right)\right| \leq C, \quad t \geq 0 .
$$

It follows from $(1.5),(1.6)_{1},(1.8)$, and $(2.3)_{1}$ that

$$
\left|u\left(x_{2}(t)-0, t\right)-u\left(x_{2}(t)+0, t\right)\right| \leq C e^{-\beta^{\prime} t}, \quad t \geq 0,
$$

and

$$
\left|\frac{r+s}{2}\left(x_{2}(t)-0, t\right)\right| \leq C e^{-\beta^{\prime} t}, \quad t \geq 0
$$

for some positive constant $\beta^{\prime}>0$.

Conditions (2.32), (2.4), (2.10), and Lemma 2.2 yield

$$
\left|r_{t}\left(x_{2}(t)-0, t\right)\right|+\left|s_{t}\left(x_{2}(t)-0, t\right)\right| \leq C e^{-\beta_{1}^{\prime} t}, t \geq 0, \quad \text { for some positive } \beta_{1}^{\prime}>0 \text {. }
$$

Hence

$$
\left(\left|u_{t}\right|+\left|u_{x}\right|+\left|v_{t}\right|+\left|v_{x}\right|\right)\left(x_{2}(t)-0, t\right) \leq C e^{-\beta_{2}^{\prime}(t)}, t \geq 0, \quad \text { for some } \beta_{2}^{\prime}>0 .
$$

Similarly, it can be obtained that

$$
\left(\left|u_{t t}\right|+\left|u_{t x}\right|+\left|u_{x x}\right|+\left|v_{t t}\right|+\left|v_{t x}\right|+\left|v_{x x}\right|\right)\left(x_{2}(t)-0, t\right) \leq C, \quad t \geq 0 .
$$

Differentiating (2.16) with respect to $x$, and using (2.17) and other established estimates, it can be shown by a similar approach to that used for Lemma 2.1 that

$$
\left|S_{x x x}(x, t)\right| \leq C \quad \text { for }(x, t) \in \Omega_{1} .
$$

Let

$$
y_{2}\left(x_{2}(t)-0, t\right)=:\left(v^{-\frac{\gamma+1}{4}} y_{x}\right)\left(x_{2}(t)-0, t\right)
$$

and

$$
Z(t)=: y_{2}\left(x_{2}(t)-0, t\right)
$$

where $y$ is defined in (2.12).

It is not difficult to get, from (2.16), that

$$
\begin{aligned}
\frac{d Z(t)}{d t}= & -b\left(x_{2}(t)-0, t\right) \cdot Z(t)+f_{3}\left(x_{2}(t)-0, t\right) \\
& +y_{2 x}\left(x_{2}(t)-0, t\right)\left[\dot{x}_{2}(t)-\mu\left(v\left(x_{2}(t)-0, t\right)\right)\right],
\end{aligned}
$$

where the functions $b$ and $f_{3}$ are defined in the proof of Lemma 2.1. By a similar argument to that used in Lemma 2.2, we obtain

$$
|Z(t)| \leq C e^{-\beta_{3}^{\prime} t}, \quad t \geq 0
$$

for some positive constant $\beta_{3}^{\prime}$. 
It then turns out that

$$
\left|s_{x x}\left(x_{2}(t)-0, t\right)\right| \leq C e^{-\beta_{4}^{\prime} t}, t \geq 0 \text { for some positive constant } \beta_{4}^{\prime}>0 .
$$

Due to (2.37), the relation between $r_{x x}$ and $s_{x x}$ along $x=x_{2}(t)$, mentioned before, and other obtained estimates, it can be shown that

$$
\left|r_{x x}\left(x_{2}(t)-0, t\right)\right| \leq C e^{-\beta_{5}^{\prime} t}, t \geq 0, \quad \text { for some positive constant } \beta_{5}^{\prime}>0 .
$$

Thus, it is possible to get the exponential decay rates for other second-order partial derivatives of $r$ and $s$ along $x=x_{2}(t)$, with the help of (2.4).

On the other hand, along the backward characteristic curve $x=x_{1}(t)$, it follows that

$$
\begin{gathered}
\frac{d x_{1}(t)}{d t}=-\sqrt{\gamma} v_{-}^{-\frac{\gamma+1}{2}}, \quad x_{1}(0)=0, \\
v\left(x_{1}(t), t\right) \equiv v_{-}, \quad u\left(x_{1}(t), t\right) \equiv u_{-} e^{-t} .
\end{gathered}
$$

Moreover, it is easy to see from (1.6) and the higher smoothness of $(u, v)$ in the domain $I_{1}$ that all of $v_{t}, v_{x}, v_{x x}, v_{x x x}$, etc. equal to zero along $x=x_{1}(t)$ while all of $u_{t}, u_{x}, u_{x x}$, $u_{x x x}$, etc. decay exponentially fast as $t \rightarrow+\infty$ along $x=x_{1}(t)$.

We now collect in the following lemma all of the estimates obtained so far.

LEMMA 2.3. Along the shock curve $x=x_{2}(t)$, it follows that

$$
\begin{aligned}
& \left|u\left(x_{2}(t)-0, t\right)\right|+\left|v\left(x_{2}(t)-0, t\right)-v_{+}\right| \\
& +\left(\left|u_{x}\right|+\left|u_{t}\right|+\left|u_{x x}\right|+\left|u_{x t}\right|+\left|u_{t t}\right|+\left|v_{x}\right|+\left|v_{t}\right|+\left|v_{x x}\right|+\left|v_{x t}\right|+\left|v_{t t}\right|\right)\left(x_{2}(t)-0, t\right) \\
& \leq C e^{-\beta^{*} t}, t \geq 0, \quad \text { for some positive constant } \beta^{*}>0 .
\end{aligned}
$$

A similar estimate along $x=x_{1}(t)$ can be expressed if we use $v_{-}$and $x_{1}(t)$ instead of $v_{+}$ and $x_{2}(t)-0$ in $(2.40)$ respectively.

3. Energy estimates and the main results. We compare the solution $(u, v)$ of (1.1), (1.3) with the similarity solution $v^{*}$ of (1.2) and establish the main theorem in this section.

It is known (see [DP]) that there exists a unique similarity solution $v^{*}(\eta), \eta=\frac{x}{\sqrt{t+1}}$, satisfying $v^{*}(\mp \infty)=v_{\mp}$, for the equation (1.2), namely $v^{*}(\eta)$ satisfies

$$
\left\{\begin{array}{l}
{\left[-p\left(v^{*}(\eta)\right)\right]_{\eta \eta}+\frac{1}{2} \eta v_{\eta}^{*}=0,} \\
v^{*}(\mp \infty)=v_{\mp} .
\end{array}\right.
$$

Moreover, it has been shown that $v_{\eta}^{*}(\eta)>0$ for $\eta \in \mathbb{R}$ if $v_{-}<v_{+}$, which implies

$$
v_{-}<v^{*}(\eta)<v_{+}
$$

The following estimates on $v^{*}(\eta)=v^{*}\left(\frac{x}{\sqrt{t+1}}\right)$ are needed in our analysis (see [HLI1, HLI2] for details). 
LEMma 3.1. For the similarity solution $v^{*}(\eta), \eta=\frac{x}{\sqrt{t+1}}$, it follows that

$$
\begin{gathered}
\left|v_{\eta \eta \eta}^{*}(\eta)\right|+\left|v_{\eta \eta}^{*}(\eta)\right|+\left|v_{\eta}^{*}(\eta)\right|+\left\{\begin{array}{l}
\left|v^{*}(\eta)-v_{+}\right| \text {if } \eta \geq 0 \quad \leq C\left|v_{+}-v_{-}\right| e^{-C \eta^{2}} \\
\left|v^{*}(\eta)-v_{-}\right| \text {if } \eta \leq 0
\end{array}\right. \\
\int_{-\infty}^{+\infty}\left|v_{x}^{*}(x, t)\right|^{2} d x \leq C\left(v_{+}-v_{-}\right)^{2}(t+1)^{-1 / 2} \\
\int_{-\infty}^{\infty}\left[\left|v_{t}^{*}(x, t)\right|^{2}+\left|v_{x x}^{*}(x, t)\right|^{2}\right] d x \leq C\left(v_{+}-v_{-}\right)^{2}(t+1)^{-3 / 2} \\
\int_{-\infty}^{\infty}\left|v_{x t}^{*}(x, t)\right|^{2} d x \leq C\left(v_{+}-v_{-}\right)^{2}(t+1)^{-5 / 2} \\
\int_{-\infty}^{\infty}\left|v_{x x t}^{*}(x, t)\right|^{2} d x \leq C\left(v_{+}-v_{-}\right)^{2}(t+1)^{-7 / 2} \\
\int_{-\infty}^{\infty}\left|v_{x t t}^{*}(x, t)\right|^{2} d x \leq C\left(v_{+}-v_{-}\right)^{2}(t+1)^{-9 / 2}
\end{gathered}
$$

where $v^{*}(x, t)=v^{*}\left(\frac{x}{\sqrt{t+1}}\right)$.

It follows from $(1.5),(1.6)_{1}$, and (1.8) that

$$
D_{1} t \leq x_{2}(t) \leq D_{2} t, \quad t \geq 0
$$

for some positive constants $D_{1}$ and $D_{2}$.

Take any smooth function $m_{0}(x)$ with

$$
\operatorname{supp} m_{0}(x) \subset[-1,1]
$$

and

$$
\int_{-\infty}^{\infty} m_{0}(x) d x=\int_{-1}^{1} m_{0}(x) d x=1
$$

Due to (3.9) and (1.7), it is possible to choose $T_{1}>0$ such that

$$
[-1,1] \subset\left[x_{1}\left(T_{1}\right), x_{2}\left(T_{1}\right)\right]
$$

Define

$$
\begin{aligned}
\nu\left(x_{0}, t\right)= & {\left[u\left(x_{2}(t)-0, t\right)-u^{*}\left(\frac{x_{2}(t)+x_{0}}{\sqrt{t+1}}\right)\right]+\left[u^{*}\left(\frac{x_{1}(t)+x_{0}}{\sqrt{t+1}}\right)-u_{-} e^{-t}\right] } \\
& +\left[v\left(x_{2}(t)-0, t\right)-v^{*}\left(\frac{x_{2}(t)+x_{0}}{\sqrt{t+1}}\right)\right] \dot{x}_{2}(t) \\
& +\left[v^{*}\left(\frac{x_{1}(t)+x_{0}}{\sqrt{t+1}}\right)-v_{-}\right] \dot{x}_{1}(t)
\end{aligned}
$$

where $u^{*}\left(\frac{x+x_{0}}{\sqrt{t+1}}\right)=-\left[p\left(v^{*}\left(\frac{x+x_{0}}{\sqrt{t+1}}\right)\right)\right]_{x}$.

It can be shown that $F\left(x_{0}\right)=:-\int_{T_{1}}^{+\infty} \nu\left(x_{0}, t\right) d t$ is well defined for $x_{0} \in R$. In fact, (3.3), (3.9), and Lemma 2.3 yield

$$
\left|u\left(x_{2}(t)-0, t\right)-u^{*}\left(\frac{x_{2}(t)+x_{0}}{\sqrt{t+1}}\right)\right| \leq C\left(x_{0}\right) e^{-\beta_{8} t}, \quad t \geq 0,
$$


for some positive constant $\beta_{8}>0$ and $C\left(x_{0}\right)>0$, depending only on $x_{0}$. Similarly,

$$
\begin{aligned}
& \left|v\left(x_{2}(t)-0, t\right)-v^{*}\left(\frac{x_{2}(t)+x_{0}}{\sqrt{t+1}}\right)\right| \leq\left|v\left(x_{2}(t)-0, t\right)-v_{+}\right| \\
& +\left|v^{*}\left(\frac{x_{2}(t)+x_{0}}{\sqrt{t+1}}\right)-v_{+}\right| \\
& \leq C\left(x_{0}\right) e^{-\beta_{9} t}, t \geq 0, \quad \text { for some } \beta_{9}>0 \text {, } \\
& \left|u^{*}\left(\frac{x_{1}(t)+x_{0}}{\sqrt{t+1}}\right)-u_{-} e^{-t}\right| \leq C\left(x_{0}\right) e^{-\beta_{10} t}, t \geq 0, \quad \text { for some } \beta_{10}>0, \\
& \left|v^{*}\left(\frac{x_{1}(t)+x_{0}}{\sqrt{t+1}}\right)-v_{-}\right| \leq C\left(x_{0}\right) e^{-\beta_{11} t}, t \geq 0, \quad \text { for some } \beta_{11}>0 .
\end{aligned}
$$

From (3.13)-(3.16), we know that $F\left(x_{0}\right)$ is well defined in $x_{0} \in R$.

Let

$$
G\left(x_{0}\right)=\int_{x_{1}\left(T_{1}\right)}^{x_{2}\left(T_{1}\right)}\left[v\left(x, T_{1}\right)-v^{*}\left(\frac{x+x_{0}}{\sqrt{T_{1}}}\right)\right] d x-F\left(x_{0}\right) .
$$

It can be verified, by using (1.5), (1.6), (1.8), and the facts of $v^{*}( \pm \infty)=v_{ \pm}, v_{-}<v_{+}$, and $u^{*}( \pm \infty)=0$ that

$$
\lim _{x_{0} \rightarrow-\infty} G\left(x_{0}\right)=+\infty \text { and } \lim _{x_{0} \rightarrow+\infty} G\left(x_{0}\right)=-\infty .
$$

Thus, we are able to choose $\bar{x}_{0} \in R$ such that

$$
G\left(\bar{x}_{0}\right)=0 .
$$

Let

$$
m(x, t)=m_{0}(x) h(t) \text { for any }(x, t) \in \Omega_{3}=:\left\{x_{1}(t) \leq x<x_{2}(t), t \geq T_{1}\right\},
$$

where

$$
h(t)=-\int_{t}^{+\infty} \nu\left(\bar{x}_{0}, \xi\right) d \xi
$$

and let

$$
\begin{aligned}
& w(x, t)=v(x, t)-v^{*}\left(\frac{x+\bar{x}_{0}}{\sqrt{t+1}}\right)-m(x, t), \\
& z(x, t)=u(x, t)-u^{*}\left(\frac{x+\bar{x}_{0}}{\sqrt{t+1}}\right)-\int_{-\infty}^{x} m_{t}(\xi, t) d \xi
\end{aligned}
$$

where $(x, t) \in \Omega_{3}$.

Thus, (1.1) and (1.2) imply that

$$
\left\{\begin{array}{l}
w_{t}=z_{x} \\
z_{t}+\left[p\left(v^{*}+m+w\right)-p\left(v^{*}\right)\right]_{x}+z-p\left(v^{*}\right)_{x t}=-\left[h_{t}(t)+h_{t t}(t)\right] \int_{-\infty}^{x} m_{0}(\xi) d \xi
\end{array}\right.
$$

in $\Omega_{3}$. 
Introduce

$$
\varphi(x, t)=\int_{x_{1}(t)}^{x} w(\xi, t) d \xi, \quad(x, t) \in \Omega_{3} .
$$

It follows then that

$$
\varphi_{x}(x, t)=w(x, t)
$$

and

$$
\varphi_{t}(x, t)=z(x, t)-z\left(x_{1}(t), t\right)-w\left(x_{1}(t), t\right) \dot{x}_{1}(t) .
$$

Define $\theta(t)=: z\left(x_{1}(t), t\right)+w\left(x_{1}(t), t\right) \dot{x}_{1}(t)$. It turns out that

$$
\begin{aligned}
\varphi_{t t}+ & {\left[p\left(v^{*}+m+\varphi_{x}\right)-p\left(v^{*}\right)\right]_{x}+\varphi_{t}-p\left(v^{*}\right)_{x t} } \\
& =-\left(h_{t}(t)+h_{t t}(t)\right) \int_{-\infty}^{x} m_{0}(\xi) d \xi+\left(\theta_{t}(t)-\theta(t)\right) .
\end{aligned}
$$

Denote the right-hand side of $(3.23)$ by $\Lambda(x, t)$, namely

$$
\Lambda(x, t)=:-\left(h_{t}(t)+h_{t t}(t)\right) \int_{-\infty}^{x} m_{0}(\xi) d \xi+\left(\theta_{t}(t)-\theta(t)\right)
$$

In view of Lemma 2.3, Lemma 3.1, (3.10), (3.12), and the uniform boundedness of $\dot{x}_{i}(t)$ and $\ddot{x}_{i}(t)(i=1,2)$, it can be shown that

$$
|\Lambda(x, t)|+\left|\Lambda_{t}(x, t)\right|+\left|\Lambda_{x}(x, t)\right| \leq C e^{-\beta_{12} t}, \quad \beta_{12}>0 .
$$

Denote $\varphi\left(x_{2}(t)-0, t\right)$ by $\psi(t)$. We claim that

$$
\psi(t) \equiv 0 \quad \text { for } t \geq T_{1} .
$$

In fact, it follows from (3.10)-(3.12) and (3.18) that

$$
\psi\left(T_{1}\right)=0 .
$$

Furthermore, due to (3.10)-(3.12), (3.19)-(3.22), and (1.6), it can be shown that

$$
\frac{d \psi(t)}{d t}=0, \quad t \geq T_{1}
$$

This, together with (3.26), implies (3.25).

The main result in the present paper is that

TheOREM 3.2. For the solution $(u, v)$ of $(1.1),(1.3)$ with (1.4), it follows that

$$
\begin{aligned}
& \sup _{x \in R}\left\{\left|u(x, t)-u^{*}\left(x+\bar{x}_{0}, t\right)\right|+\left|v(x, t)-v^{*}\left(x+\bar{x}_{0}, t\right)\right|\right\} \\
& \leq C(1+t)^{-1 / 2}, \quad t \geq T_{1}
\end{aligned}
$$

where $v^{*}\left(x+\bar{x}_{0}, t\right)=v^{*}\left(\frac{x+\bar{x}_{0}}{\sqrt{t+1}}\right), u^{*}\left(x+\bar{x}_{0}, t\right)=-p\left(v^{*}\left(x+\bar{x}_{0}, t\right)\right)_{x}$.

Proof. It follows from (3.3), (3.9), and (1.6) that

$$
\begin{aligned}
\sup _{x>x_{2}(t)}\left|v^{*}\left(x+\bar{x}_{0}, t\right)-v(x, t)\right| & \leq C\left|v_{+}-v_{-}\right| e^{-C\left(\frac{D_{1} t+\bar{t}_{0}}{\sqrt{t+1}}\right)^{2}} \\
& \leq C(1+t)^{-1 / 2}, \quad t \geq T_{1} .
\end{aligned}
$$


Similarly,

$$
\begin{aligned}
\sup _{x>x_{2}(t)} \mid u(x, t) & -u^{*}\left(x+\bar{x}_{0}, t\right) \mid \\
+ & \sup _{x \leq x_{1}(t)}\left\{\left|v(x, t)-v^{*}\left(x+\bar{x}_{0}, t\right)\right|+\left|u(x, t)-u^{*}\left(x+\bar{x}_{0}, t\right)\right|\right\} \\
& \leq C(1+t)^{-1 / 2}, \quad t \geq T_{1} .
\end{aligned}
$$

Thus, to prove (3.28) it is sufficient to show that

$\sup _{x_{1}(t)<x<x_{2}(t)}\left\{\left|u(x, t)-u^{*}\left(x+\bar{x}_{0}, t\right)\right|+\left|v(x, t)-v^{*}\left(x+\bar{x}_{0}, t\right)\right|\right\} \leq C(1+t)^{-1 / 2}, \quad t \geq T_{1}$.

By virtue of (3.16) and the fact that

$$
|h(t)| \leq C e^{-\beta_{13} t}, \quad \text { for some positive constant } \beta_{13}>0,
$$

we only need to show that

$$
\sup _{x_{1}(t)<x<x_{2}(t)}\left[\left|\varphi_{x}(x, t)\right|+\left|\varphi_{t}(x, t)\right|\right] \leq C(1+t)^{-1 / 2}, \quad t \geq T_{1}
$$

We prove (3.33) next.

Multiplying (3.23) by $\varphi$ and $\varphi_{t}$ respectively, then integrating the resulting equations on the region $\left\{(x, \tau) \mid x_{1}(\tau) \leq x \leq x_{2}(\tau), T_{1} \leq \tau \leq t\right\}$, integrating by parts and using (3.25) we arrive at

$$
\begin{aligned}
\int_{x_{1}(t)}^{x_{2}(t)} & \left(\varphi_{t} \cdot \varphi+\frac{1}{2} \varphi^{2}\right)(x, t) d x \\
& +\int_{T_{1}}^{t} \int_{x_{1}(\tau)}^{x_{2}(\tau)}\left[p\left(v^{*}\right)-p\left(v^{*}+\varphi_{x}\right)\right] \varphi_{x}(x, \tau) d x d \tau \\
= & \int_{T_{1}}^{t} \int_{x_{1}(\tau)}^{x_{2}(\tau)} \varphi_{t}^{2}(x, \tau) d x d \tau \\
& +\int_{T_{1}}^{t} \int_{x_{1}(\tau)}^{x_{2}(\tau)}\left[p\left(v^{*}+m+\varphi_{x}\right)-p\left(v^{*}+\varphi_{x}\right)\right] \varphi_{x}(t, \tau) d x d \tau \\
& -\int_{T_{1}}^{t} \int_{x_{1}(\tau)}^{x_{2}(\tau)} p\left(v^{*}\right)_{t} \varphi_{x}(x, \tau) d x d \tau+\int_{T_{1}}^{t} \int_{x_{1}(\tau)}^{x_{2}(\tau)} \wedge(x, \tau) \varphi(x, \tau) d x d \tau \\
& +\int_{x_{1}\left(T_{1}\right)}^{x_{2}\left(T_{1}\right)}\left[\varphi_{t} \varphi+\frac{1}{2} \varphi^{2}\right]\left(x, T_{1}\right) d x, \quad t \geq T_{1},
\end{aligned}
$$


and

$$
\begin{aligned}
\int_{x_{1}(t)}^{x_{2}(t)} & \frac{1}{2} \varphi_{t}^{2}(x, t) d x+\int_{T_{1}}^{t} \int_{x_{1}(\tau)}^{x_{2}(\tau)}\left[p\left(v^{*}+m\right)-p\left(v^{*}+m+\varphi_{x}\right)\right] \varphi_{x t}(x, \tau) d x d \tau \\
& +\int_{T_{1}}^{t} \int_{x_{1}(\tau)}^{x_{2}(\tau)} \varphi_{t}^{2}(x, \tau) d x d \tau \\
= & \int_{x_{1}\left(T_{1}\right)}^{x_{2}\left(T_{1}\right)} \frac{1}{2} \varphi_{t}^{2}\left(x, T_{1}\right) d x+\int_{T_{1}}^{t} \int_{x_{1}(\tau)}^{x_{2}(\tau)}\left[p\left(v^{*}\right)-p\left(v^{*}+m\right)\right]_{x} \varphi_{t}(x, \tau) d x d \tau \\
& +\int_{T_{1}}^{t} \int_{x_{1}(\tau)}^{x_{2}(\tau)} p\left(v^{*}\right)_{x t} \varphi_{t}(x, \tau) d x d \tau \\
& +\int_{T_{1}}^{t}\left[\frac{1}{2} \varphi_{t}^{2}\left(x_{2}(\tau)-0, \tau\right) \dot{x}_{2}(\tau)-\frac{1}{2} \varphi_{t}^{2}\left(x_{1}(\tau), \tau\right) \dot{x}_{1}(\tau)\right] d \tau \\
& +\int_{T_{1}}^{t} \int_{x_{1}(\tau)}^{x_{2}(\tau)} \Lambda(x, \tau) \varphi_{t}(x, \tau) d x d \tau, \quad t \geq T_{1} .
\end{aligned}
$$

Let

$$
q(x, t)=: \int_{0}^{\varphi_{x}}\left[p\left(v^{*}+m\right)-p\left(v^{*}+m+y\right)\right] d y .
$$

It can be easily verified that

$$
\begin{aligned}
q_{t}= & {\left[p\left(v^{*}+m\right)-p\left(v^{*}+m+\varphi_{x}\right)\right] \varphi_{x t} } \\
& +\int_{0}^{\varphi_{x}}\left\{p^{\prime}\left(v^{*}+m\right)-p^{\prime}\left(v^{*}+m+y\right)\right\}\left(v_{t}^{*}+m_{t}\right) d y .
\end{aligned}
$$

It is known that

$$
|m(x, t)| \leq C \delta, \quad\left|\varphi_{x}(x, t)\right| \leq C \delta \quad \text { for }(x, t) \in \Omega_{3}
$$

which implies

$$
v^{*}+m+\varphi_{x} \geq v_{-}-2 C \delta \geq \frac{v_{-}}{2}>0 \text { for small } \delta>0
$$

Since $p^{\prime}<0$, we have

$$
C_{2} \varphi_{x}^{2} \leq q \leq C_{1} \varphi_{x}^{2} \quad \text { for some } C_{1} \geq C_{2}>0
$$

It follows from Lemma 2.3 and (3.3) that

$$
\begin{aligned}
& \left|\varphi_{t}\left(x_{2}(t)-0, t\right)\right|+\left|\varphi_{t}\left(x_{1}(t), t\right)\right| \\
& \quad \leq C e^{-\beta_{14} t}, t \geq T_{1}, \quad \text { for some } \beta_{14}>0 .
\end{aligned}
$$

From (3.9) and (3.22), it can be shown that

$$
|\varphi(x, t)| \leq C \delta t, \quad\left|\varphi_{t}(x, t)\right| \leq C, \quad t \geq T_{1}, \quad x_{1}(t) \leq x \leq x_{2}(t) .
$$


Turning to the second term on the left-hand side of (3.35) and using integration by parts, it reads

$$
\begin{aligned}
\int_{T_{1}}^{t} \int_{x_{1}(\tau)}^{x_{2}(\tau)}\left[p\left(v^{*}+m\right)-p\left(v^{*}+m+\varphi_{x}\right] \varphi_{x t}(x, \tau) d x d \tau\right. \\
=\int_{T_{1}}^{t} \int_{x_{1}(\tau)}^{x_{2}(\tau)} q_{t} d x d \tau \\
\quad-\int_{T_{1}}^{t} \int_{x_{1}(\tau)}^{x_{2}(\tau)}\left\{\int_{0}^{\varphi_{x}}\left[p^{\prime}\left(v^{*}+m\right)-p^{\prime}\left(v^{*}+m+y\right)\right] d y\right\}\left(v_{t}^{*}+m_{t}\right)(x, \tau) d x d \tau \\
=\int_{x_{1}(t)}^{x_{2}(t)} q(x, t) d x-\int_{x_{1}\left(T_{1}\right)}^{x_{2}\left(T_{1}\right)} q\left(x, T_{1}\right) d x \\
\quad+\int_{T_{1}}^{t}\left[q\left(x_{1}(\tau), \tau\right) \dot{x}_{1}(\tau)-q\left(x_{2}(\tau)-o, \tau\right) \dot{x}_{2}(\tau)\right] d \tau \\
\quad-\int_{T_{1}}^{t} \int_{x_{1}(\tau)}^{x_{2}(\tau)}\left\{\int_{0}^{\varphi_{x}}\left[p^{\prime}\left(v^{*}+m\right)-p^{\prime}\left(v^{*}+m+y\right)\right] d y\left(v_{t}^{*}+m_{t}\right)\right\}(x, \tau) d x d \tau .
\end{aligned}
$$

In view of (3.38) and (3.13)-(3.16), it follows that

$$
\left|q\left(x_{1}(\tau), \tau\right)\right|+\left|q\left(x_{2}(\tau)-0, \tau\right)\right| \leq C \delta^{2} e^{-\beta_{15} \tau}, \tau \geq T_{1}, \quad \text { for some } \beta_{15}>0 .
$$

Due to (3.3), (3.19), and (3.20), the following holds:

$$
\begin{aligned}
& \left|\int_{T_{1}}^{t} \int_{x_{1}(\tau)}^{x_{2}(\tau)}\left\{\int_{0}^{\varphi_{x}}\left[p^{\prime}\left(v^{*}+m\right)-p^{\prime}\left(v^{*}+m+y\right)\right] d y\left(v_{t}^{*}+m_{t}\right)\right\}(x, \tau) d x d \tau\right| \\
& \quad \leq C \delta \int_{T_{1}}^{t} \int_{x_{1}(\tau)}^{x_{2}(\tau)} \varphi_{x}^{2}(x, \tau) d x d \tau .
\end{aligned}
$$

Conditions (3.24) and (3.34)-(3.43), with the help of Cauchy's inequality, yield that

$$
\int_{x_{1}(t)}^{x_{2}(t)}\left[\varphi^{2}+\varphi_{x}^{2}+\varphi_{t}^{2}\right](x, t) d x+\int_{T_{1}}^{t} \int_{x_{1}(\tau)}^{x_{2}(\tau)}\left(\varphi_{t}^{2}+\varphi_{x}^{2}\right)(x, \tau) d x d \tau \leq C,
$$

provided $\delta$ is suitably small.

Differentiate (3.23) with respect to $t$ and multiply it by $\varphi_{t t}$. Differentiate (3.23) with respect to $x$ and multiply it by $\varphi_{x t}$. Using a treatment similar to that used above to these two equalities respectively and taking account of (3.3), (3.9), Lemma 3.1, and Lemma 2.3 , it turns out that

$$
\int_{x_{1}(t)}^{x_{2}(t)}\left[\varphi_{t t}^{2}+\varphi_{x t}^{2}+\varphi_{x x}^{2}\right](x, t) d x+\int_{T_{1}}^{t} \int_{x_{1}(\tau)}^{x_{2}(\tau)}\left(\varphi_{t t}^{2}+\varphi_{x t}^{2}\right)(x, \tau) d x d \tau \leq C .
$$

Define

$$
A(t)=: \int_{x_{1}(t)}^{x_{2}(t)}\left(\varphi_{t}^{2}+\varphi_{x}^{2}\right)(x, t) d x .
$$

It can be shown by $(3.44),(3.45)$, and Lemma 2.1 that

$$
A(t) \in L^{1}\left(T_{1},+\infty\right)
$$


and

$$
\int_{T_{1}}^{+\infty}\left|\frac{d A(t)}{d t}\right| d t \leq C
$$

Conditions (3.46) and (3.47) imply that

$$
A(t) \rightarrow 0 \text { as } t \rightarrow \infty \text {. }
$$

It is known that

$$
\left|\varphi_{x}^{2}(x, t)\right| \leq 2\left(\int_{x_{1}(t)}^{x_{2}(t)} \varphi_{x}^{2} d x\right)^{1 / 2} \cdot\left(\int_{x_{1}(t)}^{x_{2}(t)} \varphi_{x x}^{2} d x\right)^{1 / 2}+\left|\varphi_{x}^{2}\left(x_{1}(t), t\right)\right| .
$$

Thus, (3.45), (3.48), and (3.49), combined with (3.16) and the fact that $m\left(x_{1}(t), t\right) \equiv 0$ for $t>T_{1}$, yield

$$
\sup _{x \in\left[x_{1}(t), x_{2}(t)\right)}\left|\varphi_{x}(x, t)\right| \rightarrow 0 \quad \text { as } t \rightarrow \infty .
$$

Similarly, it can be proved that

$$
\sup _{x \in\left[x_{1}(t), x_{2}(t)\right)}\left|\varphi_{t}(x, t)\right| \rightarrow 0 \quad \text { as } t \rightarrow \infty .
$$

We now turn to the estimates of decay rates. Instead of integrating $(3.23) \cdot \varphi_{t}$ over $\left\{(x, \tau) \mid x_{1}(\tau) \leq x \leq x_{2}(\tau), T_{1} \leq \tau \leq t\right\}$ to obtain (3.35) we integrate over $\{(x, \tau) \mid$ $\left.x_{1}(\tau) \leq x \leq x_{2}(\tau), t \leq \tau \leq T, t \geq T_{1}\right\}$ and use a similar approach as in (3.35)-(3.43) to obtain that

$$
\begin{aligned}
\int_{x_{1}(T)}^{x_{2}(T)} & \left(\frac{1}{2} \varphi_{t}^{2}+q\right)(x, T) d x \\
\leq & \int_{x_{1}(t)}^{x_{2}(t)}\left(\frac{1}{2} \varphi_{t}^{2}+q\right)(x, t) d x \\
& +C \int_{t}^{T} \int_{x_{1}(\tau)}^{x_{2}(\tau)}\left\{\varphi_{x}^{2}\left(\left|v_{t}^{*}\right|+\left|m_{t}\right|\right)+p\left(v^{*}\right)_{x t}^{2}+\left[\left(p\left(v^{*}\right)-p\left(v^{*}+m\right)\right)_{x}\right]^{2}\right\}(x, \tau) d x d \tau \\
& +C \int_{t}^{T} \tau e^{-\beta \tau} d \tau, \quad \text { for some } \beta>0 .
\end{aligned}
$$

We then integrate the above for $T_{1} \leq t \leq T$ to get

$$
\begin{aligned}
(T- & \left.T_{1}\right) \int_{x_{1}(T)}^{x_{2}(T)}\left(\frac{1}{2} \varphi_{t}^{2}+q\right)(x, T) d x \\
& \leq \int_{T_{1}}^{T} \int_{x_{1}(t)}^{x_{2}(t)}\left(\frac{1}{2} \varphi_{t}^{2}+q\right)(x, t) d x d t+C \int_{T_{1}}^{T}\left(\int_{t}^{T} l(\tau) d \tau\right) d t \\
& +C \int_{T_{1}}^{T}\left(\int_{t}^{T} \tau e^{-\beta \tau} d \tau\right) d t
\end{aligned}
$$


where

$$
l(\tau)=\int_{x_{1}(\tau)}^{x_{2}(\tau)}\left\{\varphi_{x}^{2}\left(\left|v_{t}^{*}\right|+\left|m_{t}\right|\right)+p\left(v^{*}\right)_{x t}^{2}+\left[\left(p\left(v^{*}\right)-p\left(v^{*}+m\right)\right)_{x}\right]^{2}\right\}(x, \tau) d x .
$$

Integrating by parts gives

$$
\int_{T_{1}}^{T}\left(\int_{t}^{T} l(\tau) d \tau\right) d t=\int_{T_{1}}^{T}\left(t-T_{1}\right) l(t) d t
$$

From (3.3)-(3.6), the definition of $m$, and (3.44), noting that $v_{t}^{*}=-\frac{1}{2} \eta v_{\eta}^{*}(t+1)^{-1}$, we can show

$$
\int_{T_{1}}^{T}\left(t-T_{1}\right) l(t) d t \leq C
$$

Similarly to (3.51), we have

$$
\int_{T_{1}}^{T}\left(\int_{t}^{T} \tau e^{-\beta \tau} d \tau\right) d t=\int_{T_{1}}^{T} t\left(t-T_{1}\right) e^{-\beta t} d t \leq C .
$$

Conditions (3.44) and (3.50)-(3.53) imply that

$$
\int_{x_{1}(T)}^{x_{2}(T)}\left(\frac{1}{2} \varphi_{t}^{2}+q\right)(x, T) d x \leq \frac{C}{T-T_{1}} .
$$

With a similar approach as above we can arrive at

$$
\int_{x_{1}(\tau)}^{x_{2}(\tau)}\left[\varphi_{t x}^{2}+\varphi_{x x}^{2}\right](x, T) d x \leq \frac{C}{T-T_{1}}
$$

From the arbitrariness of $T$, and (3.49), it can be shown that

$$
\sup _{x \in\left[x_{1}(t), x_{2}(t)\right)}\left[\left|\varphi_{x}(x, t)\right|+\left|\varphi_{t}(x, t)\right|\right] \leq C(1+t)^{-1 / 2},
$$

which completes the proof of (3.33). Theorem 3.2 then follows.

\section{REFERENCES}

[CH] T. Chang and L. Hsiao, The Riemann Problem and Interaction of Waves in Gas Dynamics, Pitman Monographs and Surveys in Pure and Applied Math., vol. 41, Longman, 1989

[DD] C. M. Dafermos and R. DiPerna, The Riemann problem for certain classes of hyperbolic systems of conservation laws, J. Differential Equations 20, 90-114 (1976)

[DP] C. J. Van Duyn and L. A. Peletier, A class of similarity solutions of the nonlinear diffusion equation, Nonlinear Analysis 1, 223-233 (1977)

[HLI1] L. Hsiao and T. P. Liu, Convergence to nonlinear diffusion waves for solutions of a system of hyperbolic conservation laws with damping, Comm. Math. Phys. 143, 599-605 (1992)

[HLI2] L. Hsiao and T. P. Liu, Nonlinear diffusive phenomena of nonlinear hyperbolic systems, Chinese Ann. Math. Ser. B 14, 465-480 (1993)

[HLU] L. Hsiao and T. Luo, Nonlinear diffusive phenomena of solutions for the system of compressible adiabatic flow through porous media, J. Differential Equations 125, 329-365 (1996)

[HT1] L. Hsiao and S. Q. Tang, Construction and qualitative behavior of solutions for a system of nonlinear hyperbolic conservation laws with damping, Quart. Appl. Math. 53, 487-505 (1995)

[HT2] L. Hsiao and S. Q. Tang, Construction and qualitative behavior of the solution of the perturbated Riemann problem for the system of one-dimensional isentropic flow with damping, J. Differential Equations 123, 480-503 (1995)

[LI] Tai-ping Liu, Existence and uniqueness theorems for Riemann problems, Trans. Amer. Math. Soc. 213, 375-382 (1975)

[SM] J. Smoller, Shock Waves and Reaction-Diffusion Equations, Springer-Verlag, 1980 